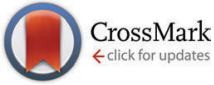

Cite this: Phys. Chem. Chem. Phys., 2014, 16, 24610

Received 2nd September 2014 Accepted 30th September 2014

DOI: 10.1039/c4cp03946g

www.rsc.org/pccp

\section{Tin doping speeds up hole transfer during light-driven water oxidation at hematite photoanodes $\dagger$}

\author{
Halina K. Dunn, $\neq^{* a}$ Johann M. Feckl, $\neq^{a}$ Alexander Müller, ${ }^{a}$ Dina Fattakhova-Rohlfing, ${ }^{a}$ \\ Samuel G. Morehead, ${ }^{a}$ Julian Roos, ${ }^{a}$ Laurence M. Peter, ${ }^{b}$ Christina Scheu $^{a}$ and \\ Thomas Bein*a
}

\begin{abstract}
Numerous studies have shown that the performance of hematite photoanodes for light-driven water splitting is improved substantially by doping with various metals, including tin. Although the enhanced performance has commonly been attributed to bulk effects such as increased conductivity, recent studies have noted an impact of doping on the efficiency of the interfacial transfer of holes involved in the oxygen evolution reaction. However, the methods used were not able to elucidate the origin of this improved efficiency, which could originate from passivation of surface electron-hole recombination or catalysis of the oxygen evolution reaction. The present study used intensity-modulated photocurrent spectroscopy (IMPS), which is a powerful small amplitude perturbation technique that can de-convolute the rate constants for charge transfer and recombination at illuminated semiconductor electrodes. The method was applied to examine the kinetics of water oxidation on thin solution-processed hematite model photoanodes, which can be Sn-doped without morphological change. We observed a significant increase in photocurrent upon Sn-doping, which is attributed to a higher transfer efficiency. The kinetic data obtained using IMPS show that Sn-doping brings about a more than tenfold increase in the rate constant for water oxidation by photogenerated holes. This result provides the first demonstration that Sn-doping speeds up water oxidation on hematite by increasing the rate constant for hole transfer.
\end{abstract}

\section{Introduction}

The photoelectrochemical splitting of water into hydrogen and oxygen under solar irradiation holds the promise of providing a vital fuel for a future low-carbon energy economy. In order to reach competitive efficiencies for hydrogen production, tandem cell architectures will be required, ${ }^{1}$ for example by connecting appropriate n-type and p-type semiconductors in optical series. ${ }^{2}$ This has sparked intensive research into semiconductor materials able to perform one of the half-reactions in water splitting. Metal oxide semiconductors are promising materials for this application

\footnotetext{
${ }^{a}$ Department of Chemistry and Center for NanoScience (CeNS), Ludwig-Maximilians-Universität München, Butenandtstr. 11, D-81377 Munich, Germany.E-mail: bein@lmu.de, halina.dunn@cup.lmu.de

${ }^{b}$ Department of Chemistry, University of Bath, BA2 7AY, Bath, UK

$\dagger$ Electronic supplementary information (ESI) available: Further synthetic details, powder XRD, SEM images, absorbance, dark cyclic voltammetry data, cyclic voltammetry of $20 \%$ Sn sample under AM 1.5 , electron diffraction data obtained in TEM, Mott-Schottky analysis, determination of $W_{\text {sc }}$ from Mott-Schottky and Gärtner analysis, cyclic voltammograms recorded under chopped illumination, EQE, IQE, geometrical estimation of the roughness of the films. See DOI: 10.1039/ c4cp03946g

\# These authors contributed equally.
}

owing to their relative low cost, ease of preparation and stability. ${ }^{3}$ However, the sluggish kinetics of the light-driven oxygen and hydrogen evolution reactions (OER and HER, respectively) compared with recombination of electrons and holes typically limit the efficiency of metal oxide photoelectrodes. The problem of recombination is evident from the characteristic "spike and overshoot" in the transient photocurrent response to chopped illumination, which has been observed in $\mathrm{Fe}_{2} \mathrm{O}_{3}, \mathrm{BiVO}_{4}, \mathrm{Cu}_{2} \mathrm{O}$ and $\mathrm{WO}_{3}$ photoelectrodes during HER and OER. ${ }^{4-7}$

Hematite, or $\alpha-\mathrm{Fe}_{2} \mathrm{O}_{3}$, is one of the most widely studied photoanode materials for the OER, owing to its chemical stability in basic media, abundance, visible light absorption and suitable valence band energy. Significant improvements in the performance of hematite photoanodes have been achieved through nanostructuring, which has helped to overcome the trade-off that exists between sufficient light absorption and carrier collection (due to hematite's indirect bandgap and poor hole-mobility). ${ }^{8,9}$ Doping $\S$ of hematite with additives such as $\mathrm{Sn}^{10-14} \mathrm{Si}^{8,15-18}$

$\S$ The term 'doping' is widely used in the water splitting literature, and we therefore use it here whilst noting that the levels of inclusion of 'dopants' are generally many orders of magnitude higher than those encountered in classical semiconductor physics. 
$\mathrm{Ti}^{10,19-23} \mathrm{Pt}^{24,25} \mathrm{Cr}^{26} \mathrm{Mo}^{26} \mathrm{Zn}^{27}$ and $\mathrm{I}^{28}$ enhances the performance of hematite for the light-driven OER. Several studies have attributed the effect of such dopants to changes in bulk hematite properties such as conductivity, ${ }^{10,11,13,16,18,19,29,30}$ or crystallinity, ${ }^{8}$ but very few studies have explored other possible beneficial roles that dopant atoms may play in the processes involved in lightdriven oxygen evolution. However, recent work by Zandi et al. ${ }^{31}$ and Chemelewski et al. ${ }^{17}$ has not only shown that Ti and Si do not act as an electrical dopant in their materials, but also that the efficiency of interfacial hole transfer to take part in the OER (or "transfer efficiency") is improved by doping.

The hole transfer efficiency, which is of the order of just $25 \%$ under standard operating conditions (at $1.23 \mathrm{~V}$ vs. RHE under AM 1.5 illumination) for benchmark hematite photoanodes, ${ }^{32}$ can be assessed by comparing the photocurrent of a photoanode in the presence and absence of a hole scavenger ${ }^{32}$ or by analysis of photocurrent transients to chopped illumination. ${ }^{7}$ While these methods are equivalent in the evaluation of the transfer efficiency, only the latter allows the distinction between a catalytic effect and a passivation of surface recombination (this is discussed in detail below). The sluggish kinetics of the multistep (4-hole) oxygen evolution reaction leads to a large build-up of photogenerated holes that are vulnerable to recombination with electrons. The competition between recombination and transfer thus lowers the efficiency of hole-transfer to the solution phase. The hole transfer efficiency can therefore be improved by speeding up interfacial hole transfer by catalysis ${ }^{33-35}$ and by suppressing surface recombination. ${ }^{7,36}$ In spite of the obvious importance of the kinetics of interfacial charge transfer in this context, only a few studies have measured the rate constants for reactions involving photogenerated holes. ${ }^{7}$

The lack of kinetic information arises from the fact that methods commonly used to measure rate constants for electron transfer at metal electrodes are not applicable to reactions involving photogenerated minority carriers at illuminated semiconductor electrodes. The reason for this is that the rate constants cannot be changed simply by altering the applied potential. Variations in potential appear predominantly across the space charge region in the semiconductor rather than across the Helmholtz layer, so in the ideal case at least, the rate constants remain unperturbed. The rate (rather than rate constant) of reactions involving photogenerated holes can be changed by altering the illumination intensity. This gives rise to a family of experimental methods that are analogues of conventional electrochemical techniques, with potential perturbation replaced by perturbation of light intensity. ${ }^{37}$ So, for example, the potential step method to determine rate constants at metal electrodes corresponds to the light step method for determining rate constants at illuminated photoelectrodes. The electrochemical impedance method for metal electrodes corresponds to intensity modulated photocurrent spectroscopy (IMPS), which is based on a small ac perturbation of the light intensity. ${ }^{38-40}$ Although these methods have been known for some time, surprisingly few studies of light-driven water splitting have made use of them to understand the influence of interfacial kinetics on efficiency. Here we illustrate the power of the approach by an IMPS study of the influence of tin-doping on light-driven water splitting at hematite electrodes.
In practice, one of the most widely encountered effects of doping of hematite layers is a modification of the nanostructure morphology, leading in many cases to a reduction in feature size and thus to an increase in surface area. $8,11,13,14,16,20,23,26,29,41$ Although this effect may be partly responsible for the reported performance improvement, it complicates the task of uncovering other possible roles played by the dopant atoms. In order to circumvent this problem, we have developed a simple solutionprocessed route to fabricate model systems in which up to 3.2 atomic \% Sn can be incorporated into thin nanostructured hematite films with only minimal morphological change. The films are about $50 \mathrm{~nm}$ thick, and produce $0.06 \mathrm{~mA} \mathrm{~cm}^{-2}$ at $1.23 \mathrm{~V}$ vs. RHE under AM 1.5 illumination. The extremely thin nature of these films leads to a low light harvesting efficiency, and consequently modest performance. However, they make good model systems because their internal quantum efficiency (IQE) under standard conditions is comparable to that of benchmark hematite prepared by atmospheric pressure chemical vapour deposition, APCVD, ${ }^{32}$ (see ESI $\dagger$ for details). Indeed, $400 \mathrm{~nm}$ thick films of our material give $0.38 \mathrm{~mA} \mathrm{~cm}^{-2}$ at $1.23 \mathrm{~V} v s$. RHE under AM 1.5 illumination, which is very close to the photocurrent of benchmark solution-processed Sn-doped hematite photoanodes. ${ }^{14}$ Nonetheless, we chose to use the thin films for mechanistic studies, since these constitute the simplest model system.

In line with other studies, ${ }^{10-14}$ we found that Sn-doping our hematite photoanodes significantly enhances the photocurrent. This improvement in performance can be attributed to a concomitant increase in the transfer efficiency in good agreement with recent work by Zandi et al. ${ }^{31}$ and Chemelewski et al. ${ }^{17}$ In principle, the improvement could be explained either by a higher rate constant for the transfer of holes across the interface, or by suppression of surface electron-hole recombination. In order to discover which explanation holds in the present case, the rate constants for surface recombination and charge transfer were de-convoluted using intensity-modulated photocurrent spectroscopy (IMPS), which showed that Sn-doping increases the rate constant for hole-transfer by more than an order of magnitude. The material composition was characterized by analytical transmission electron microscopy (TEM), which probes the degree of Sn-incorporation in the bulk of the hematite crystallites and provides insights into the spatial distribution of Sn. It emerged that $\mathrm{Sn}$-atoms are incorporated into the hematite structure without phase separation or formation of tin oxide clusters, but that the tin atoms are preferentially distributed in the near-surface regions of the hematite nanoparticles, resulting in a core-shell type structure. The results of our study thus provide the first proof that doping hematite can speed up the interfacial reaction of photogenerated holes, which is one of the fundamental limitations of hematite and other photoelectrodes for light driven water splitting.

\section{Theory}

The external quantum efficiency, $\operatorname{EQE}(\lambda)$, of light-driven water oxidation taking place at bulk semiconductor electrolyte junctions depends on the product of the efficiencies of light harvesting, 
$\eta_{\mathrm{LH}}(\lambda)$, charge separation, $\eta_{\mathrm{sep}}(\lambda)$, and hole-transfer to the electrolyte, $\eta_{\text {trans }}$, the first two being functions of the light wavelength, $\lambda$.

$$
\operatorname{EQE}(\lambda)=\eta_{\mathrm{LH}}(\lambda) \eta_{\mathrm{sep}}(\lambda) \eta_{\text {trans }}
$$

In the absence of light scattering and internal reflection, the light harvesting efficiency can be calculated from the wavelength-dependent absorption coefficient, $\alpha(\lambda)$, and the film thickness, $d$.

$$
\eta_{\mathrm{LH}}(\lambda)=1-\mathrm{e}^{-\alpha(\lambda) d}
$$

For a planar electrode geometry, the electron-hole separation efficiency $\eta_{\text {sep }}(\lambda)$ can be calculated using the Gärtner equation $^{42}$ (see below) if the width of the space charge region, $W_{\text {sc }}$, and the hole diffusion length, $L_{\mathrm{p}}$, are known. However, this calculation will not be correct if substantial recombination takes place in the space charge region (in which case $\eta_{\text {sep }}$ is lower) or if the electrode is nanostructured (see $\mathrm{ESI} \dagger$ ). For this reason, we derive $\eta_{\text {sep }}(\lambda)$ from the measured external quantum efficiency using the light harvesting efficiency calculated from the absorption spectrum and the transfer efficiency derived using the rate constants for hole transfer and recombination obtained from IMPS or photocurrent transient measurements.

Hematite photoanodes respond to chopped illumination with a characteristic "spike and overshoot" photocurrent transient. ${ }^{43-45}$ This transient response is typical for systems with a large degree of surface electron-hole recombination. ${ }^{7,45}$ When the light is switched on, holes generated in the space charge region are swept rapidly towards the semiconductor electrolyte junction. Due to the slow kinetics of the 4-hole oxidation of water to molecular oxygen, the concentration of holes builds up considerably at the interface until the rate of arrival of holes is balanced in the steady state by the rates of charge transfer and recombination. Since surface recombination leads to a flux of electrons towards the surface, the resulting photocurrent transient is the sum of the hole and electron contributions. The instantaneous photocurrent measured when the illumination is switched on corresponds to a charging or displacement current due to the initial movement of photo-generated holes towards the surface. By contrast, the steady-state photocurrent corresponds to the flux of holes that are transferred successfully to the electrolyte without undergoing recombination with electrons at the surface. It follows that the ratio of the steady state photocurrent to the instantaneous photocurrent, $j_{\mathrm{ss}} / j_{(t=0)}$, is a measure of the efficiency of hole-transfer from the electrode to the electrolyte. This situation has been modelled in terms of the surface concentration of holes using a simple phenomenological approach. ${ }^{38,39}$ Assuming that both hole transfer and recombination are pseudo-first order in the surface hole concentration, the transfer efficiency can also be expressed in terms of the phenomenological first order rate constants of hole transfer, $k_{\text {trans }}$, and recombination, $k_{\text {recc }}$.

$$
\eta_{\text {trans }}=\frac{j_{\text {ss }}}{j_{t=0}}=\frac{k_{\text {trans }}}{k_{\text {trans }}+k_{\text {rec }}}
$$

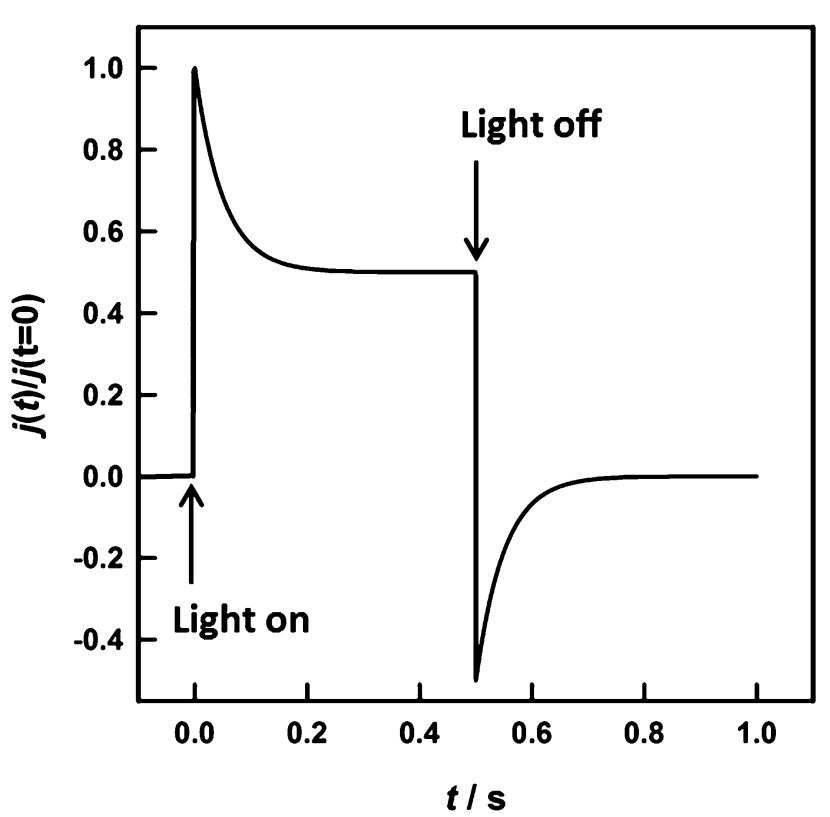

Fig. 1 Normalized transient photocurrent response calculated for $k_{\mathrm{tr}}=$ $k_{\text {rec }}=10 \mathrm{~s}^{-1}$. The decay time constant in this case is $\left(k_{\mathrm{rec}}+k_{\mathrm{tr}}\right)^{-1}=50 \mathrm{~ms}$ and the hole transfer efficiency $=k_{\mathrm{tr}} /\left(k_{\mathrm{tr}}+k_{\mathrm{rec}}\right)=0.5$, so that the steady state current is half of the instantaneous current.

An example of the type of predicted transient photocurrent response is shown in Fig. 1.

In principle, the exponential decay of the current towards the steady state, which is characterized by the time constant $\left(k_{\text {trans }}+k_{\text {rec }}\right)^{-1}$, can be analysed, and then $k_{\text {trans }}$ and $k_{\text {rec }}$ can be separated using eqn (3). In practice, however, it is more convenient to determine the time constant using small amplitude frequency-resolved measurements such as IMPS $^{7}$ or photoelectrochemical impedance spectroscopy (PEIS). ${ }^{46}$ The IMPS method involves small amplitude $(<10 \%)$ variable frequency sinusoidal modulations of the light intensity about a dc value. The resulting phase and amplitude of the photocurrent are recorded as a function of frequency, and the results are displayed in the complex plane. ${ }^{47,48}$ The imaginary component of the photocurrent reaches a maximum when the frequency, $\omega_{\max }$, matches the characteristic relaxation constant of the system, i.e. the same time constant $\left(k_{\text {trans }}+k_{\text {rec }}\right)^{-1}$ seen in the exponential decay of the transient photocurrent.

$$
\omega_{\text {max }}=k_{\text {trans }}+k_{\text {rec }}
$$

The high and low frequency intercepts of the IMPS response in the complex plane correspond respectively to the instantaneous and steady state photocurrents seen in Fig. 1, and their ratio is therefore given by eqn (3). The main advantage of the IMPS technique is that it involves a small amplitude modulation, so that changes in band bending induced by illumination are minimized (the effect on the IMPS response of lightinduced modulation of band bending has been considered elsewhere $^{49}$ ). By contrast, large changes in band bending may occur with chopped illumination (this probably explains the 


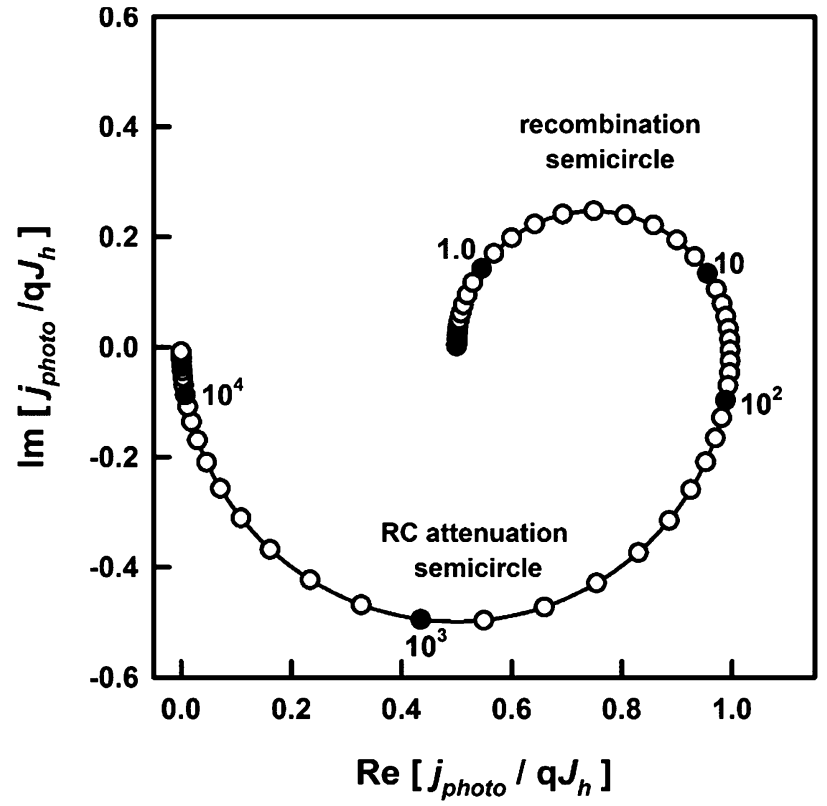

Fig. 2 IMPS response predicted for $k_{\text {rec }}=k_{\text {trans }}=10 \mathrm{~s}^{-1}, C_{\mathrm{sc}}=1 \mu \mathrm{F} \mathrm{cm} \mathrm{cm}^{-2}$, $R_{\text {ser }}=20 \Omega$. The response is normalized to the hole current, $q J_{h}$, generated by collection of holes in the space charge region. The radial frequency corresponding to the maximum of the upper recombination semicircle is equal to $k_{\text {trans }}+k_{\text {rec }}$ and the normalized low frequency intercept is equal to $k_{\text {trans }} /\left(k_{\text {trans }}+k_{\text {rec }}\right)$, which corresponds to the ratio of the steady state current to the instantaneous current in Fig. 1.

lack of symmetry between transient 'on' and 'off' responses in Fig. 5a). The IMPS response is also attenuated by the RC time constant determined by the product of the series resistance, $R_{\text {series}}$, and the space charge capacitance, $C_{\mathrm{sc}} \cdot$ ๆ This attenuation gives rise to a high semicircle in the opposite quadrant with a maximum circular frequency equal to the product $R_{\mathrm{series}} C_{\mathrm{sc}}$, which allows estimation of the space charge capacitance, $C_{\mathrm{sc}}$, if the series resistance is known. An example of the IMPS response predicted for the same values of $k_{\text {trans }}$ and $k_{\text {rec }}$ as those used for the transient in Fig. 1 is shown in Fig. 2.

As noted above, this interpretation of photocurrent transients and IMPS is valid for semiconductors with a well-defined depletion layer at the interface with the electrolyte. To be applicable to structured semiconductors, such as those studied here, $W_{\mathrm{sc}}$ should be smaller than the average feature size. One method to determine the width of the depletion region is through the measurement of the electrode capacitance. The flat band potential, $V_{\mathrm{fb}}$, and donor density, $N_{\mathrm{d}}$, derived from the MottSchottky relationship ${ }^{50}$ (eqn (5)), are then used to calculate values of $W_{\mathrm{sc}}$ as a function of applied potential (eqn (6)), where $\varepsilon_{\mathrm{r}}$ is the relative permittivity, $\varepsilon_{0}$ is the permittivity of free space, $A$ is the electrode area, $V$ is the applied potential, $q$ is the elementary charge, $k_{\mathrm{B}}$ is Boltzmann's constant and $T$ is the temperature.

$$
\frac{1}{C_{\mathrm{sc}}^{2}}=\frac{2}{\varepsilon_{\mathrm{r}} \varepsilon_{0} N_{\mathrm{d}} A^{2}}\left[\left(V-V_{\mathrm{fb}}\right)-\frac{k_{\mathrm{B}} T}{q}\right]
$$

T The RC time constant also determines the rise time of the transient photocurrent. This has not been considered in the calculation of Fig. 1.

$$
W_{\mathrm{sc}}=\left(\frac{2\left(V-V_{\mathrm{fb}}\right) \varepsilon_{\mathrm{r}} \varepsilon_{0}}{q N_{\mathrm{d}}}\right)^{1 / 2}
$$

Another method to estimate the width of the space charge layer in illuminated semiconductor electrolyte junctions under reverse bias involves using the Gärtner equation. ${ }^{42}$ This equation applies if holes are consumed so rapidly at the interface that there are no recombination losses (this is the case if a fast redox system is used to capture holes). For materials with very small hole-diffusion lengths, $L_{\mathrm{p}}$, such as hematite, only carriers generated in the space charge layer contribute to the photocurrent, simplifying the Gärtner expression.

$$
\operatorname{EQE}(\lambda)=1-\frac{\mathrm{e}^{-\alpha(\lambda) W_{\mathrm{sc}}}}{1+\alpha(\lambda) L_{\mathrm{p}}} \approx 1-\mathrm{e}^{-\alpha(\lambda) W_{\mathrm{sc}}}
$$

However, in the case of the light driven OER, some fraction of the holes reaching the surface is lost by surface recombination so that the EQE is lower than predicted by eqn (7). If we take the non-unity transfer efficiency into account, the simplified Gärtner equation can be rearranged to give the width of the space charge region.

$$
W_{\mathrm{sc}}=-\frac{1}{\alpha(\lambda)} \ln \left(1-\frac{\mathrm{EQE}}{\eta_{\text {trans }}}\right)
$$

\section{Experimental}

Synthetic route for the incorporation of Sn into mesoporous hematite electrodes

Hematite precursor solutions were prepared according to the following procedure. $0.630 \mathrm{~g}(1.56 \mathrm{mmol}) \mathrm{Fe}\left(\mathrm{NO}_{3}\right)_{3} \cdot 9 \mathrm{H}_{2} \mathrm{O}$ was dissolved in a solution of the block copolymer Pluronic P123 $(0.25 \mathrm{~g})$ in $10 \mathrm{~mL}$ tert-butanol under sonication for 15 minutes. $2.5 \mathrm{~mL}$ water (Millipore) was then added, forming a dark red solution. The solution was stirred at room temperature overnight resulting in a light brown dispersion of iron oxide $\left(\mathrm{Fe}_{2} \mathrm{O}_{3}\right)$. The synthesis is a development of the protocol described by Redel et al. ${ }^{51}$ However, these authors did not use any surfactant, and their synthesis led to formation of a two-phase mixture of hematite and maghemite, in the ratio of $65: 35$. By contrast, addition of the surfactant yields phase-pure hematite.

For the preparation of the Sn-containing hematite, $\mathrm{Sn}(\mathrm{OAc})_{4}$ was added to the solution described above, see Table $\mathrm{S} 1$ in the $\mathrm{ESI} \dagger$ for further details. The desired amounts of $\mathrm{Sn}(\mathrm{OAc})_{4}$ were first dispersed under vigorous stirring for $5 \mathrm{~h}$ followed by 15 min sonication in the above mixture of Pluronic P123 and tert-butanol. The remaining steps of the synthesis then followed those described above for pure hematite. It is important to note that throughout the following text, the $\mathrm{Sn}$ : Fe-ratio refers to the atomic ratio of the two elements in the precursor solutions, unless stated otherwise.

After cleaning the FTO glass (Pilkington TEC 15 Glass $^{\mathrm{TM}}$, $2.5 \times 1.5 \mathrm{~cm}$ ) by sequential sonication for $15 \mathrm{~min}$ each in detergent $(1 \mathrm{~mL}$ Extran in $50 \mathrm{~mL}$ Millipore water), water 
(Millipore) and ethanol, the substrates were dried and masked with Scotch Tape on the conducting side to retain a noncovered area of $1.5 \times 1.5 \mathrm{~cm}$. The backs of the substrates were completely masked to avoid contamination during the spincoating procedure.

Before spin-coating, the fresh solutions were filtered through a $220 \mathrm{~nm}$ syringe filter (Sartorius Minisart cellulose acetate membrane) to remove agglomerates, ensuring the preparation of homogeneously smooth films. The masked substrates were covered with $100 \mu \mathrm{L}$ of solution and spun at $1000 \mathrm{rpm}$ for 30 seconds. To remove the surfactant and crystallize the material, the samples were calcined in air in a laboratory oven ( 3 hour ramp to $600{ }^{\circ} \mathrm{C}, 30 \mathrm{~min}$ dwell time), resulting in films of about $50 \mathrm{~nm}$ thickness. To obtain thicker films, the films were dried for 5 minutes at $60{ }^{\circ} \mathrm{C}$ and the spin-coating step was repeated. Powders for X-ray diffraction (XRD) and TEM analysis were obtained by scraping material off the substrate with a razor blade.

\section{Thin film characterization}

XRD measurements were performed on a STOE powder diffractometer in transmission geometry $\left(\mathrm{Cu}-\mathrm{K} \alpha_{1}, \lambda=1.5406 \AA\right)$ equipped with a position-sensitive Mythen-1K detector. Scanning electron microscopy (SEM) was performed on a JEOL JSM$6500 \mathrm{~F}$ scanning electron microscope equipped with a field emission gun. Material was scraped from the substrate, and deposited on a copper grid with a carbon film for TEM analysis. The analysis was carried out on a FEI Titan 80-300 (S)TEM with a Fischione Instruments (Model 3000) high angle annular dark field (HAADF) detector and an EDAX energy-dispersive X-ray spectroscopy (EDX) detector. All measurements were conducted at an acceleration voltage of $300 \mathrm{kV}$.

The UV-visible transmission and reflection of the mesoporous thin films on FTO-coated glass substrates was measured with a Perkin Elmer Lambda 1050 UV/Visible/NIR spectrophotometer equipped with an integrating sphere. For transmission measurements, the sample was placed directly outside the integrating sphere with the film facing inwards, and for reflection measurements, the sample was placed directly after the integrating sphere with the glass substrate facing the incoming beam. The Naperian absorbance spectra of the hematite layers $\mathrm{Abs}_{\mathrm{H}}$ were calculated from the wavelength dependent transmission of the FTO substrate and hematite-coated substrate, $T_{\mathrm{S}}$ and $T_{\mathrm{S}+\mathrm{H}}$, respectively, and reflection of the substrate and hematite coated sample, $R_{\mathrm{S}}$ and $R_{\mathrm{S}+\mathrm{H}}$, according to the following formula, which is derived in ref. 52 .

$$
\operatorname{Abs}_{\mathrm{H}}=\ln \left(\frac{\frac{T_{\mathrm{S}+\mathrm{H}}}{T_{\mathrm{S}}}}{1-\frac{R_{\mathrm{S}+\mathrm{H}}-R_{\mathrm{S}}}{T_{\mathrm{S}}^{2}}}\right)
$$

\section{Photoelectrochemical characterization}

Hematite photoelectrodes were masked with a PTFE-coated glass fibre adhesive tape leaving a circular area of $1 \mathrm{~cm}$ in diameter exposed to a $0.1 \mathrm{M} \mathrm{NaOH}$ aqueous electrolyte.
Electrochemical measurements were carried out in a cubic optical grade glass cell (Hellma Analytics) using a $\mu$-Autolab III potentiostat (Metrohm) equipped with an FRA 2 impedance analyser connected to a saturated $\mathrm{Ag} / \mathrm{AgCl}$ reference electrode (Sigma Aldrich, $0.197 \mathrm{~V}$ vs. SHE) and a Pt mesh counter electrode. Electrode potentials versus the reversible hydrogen electrode, $V_{\mathrm{RHE}}$, were calculated from those measured at $\mathrm{pH} 13$ versus the $\mathrm{Ag} / \mathrm{AgCl}$ electrode, $V_{\mathrm{Ag} / \mathrm{AgCl}}$, according to

$$
\left(\frac{V_{\mathrm{RHE}}-V_{\mathrm{Ag} / \mathrm{AgCl}}}{V}\right)=0.197+0.059 \mathrm{pH}
$$

The light intensity was measured at the position of the electrode inside the cell using a $4 \mathrm{~mm}^{2}$ photodiode, which had been calibrated against a certified Fraunhofer ISE silicon reference cell equipped with a KG5 filter.

The current-voltage characteristics of the films were obtained by scanning from negative to positive potentials in the dark or under illumination with a $20 \mathrm{mV} \mathrm{s}^{-1}$ sweep rate. Illumination, provided either by a high-power light emitting diode (LED, Thorlabs, $455 \mathrm{~nm}$ ) or by a solar simulator (AM1.5G. Solar Light Model 16S) at $100 \mathrm{~mW} \mathrm{~cm}^{-2}$, was incident through the FTO-coated glass substrate. For external quantum efficiency (EQE) measurements, chopped monochromatic light (chopping frequency $2 \mathrm{~Hz}$ ) was provided by a $150 \mathrm{~W}$ xenon lamp in combination with a monochromator and order-sorting filters. The cell was biased close to $1.2 \mathrm{~V}$ vs. RHE under simulated solar irradiation to ensure realistic operating conditions. The current recorded by the Autolab potentiostat was output to a lock-in amplifier synchronized to the chopper frequency.

Photocurrent transients were used to estimate the transfer efficiency of holes to the solution phase. ${ }^{7}$ The high power light emitting diode, LED, was switched on and off every $500 \mathrm{~ms}$. The hematite electrodes were held at a given potential, and the transient current was sampled at $0.1 \mathrm{~ms}$ intervals. This fast sampling allowed the instantaneous current to be determined. In cases where the current transient had not reached a steady state value after $500 \mathrm{~ms}$, additional photocurrent transients were recorded with $5 \mathrm{~s}$ on/off times, sampled at $1 \mathrm{~ms}$ intervals. Electrochemical impedance spectroscopy (EIS) was carried out in the dark at applied potentials at which no significant dark current flows (between 0.75 and $1.5 \mathrm{~V} v$ s. RHE), and the potential was modulated by $10 \mathrm{mV}$ at frequencies ranging logarithmically from $100 \mathrm{kHz}$ to $1 \mathrm{~Hz}$. Intensity modulated photocurrent spectroscopy (IMPS) was carried out using a PGSTAT302N Autolab (Metrohm), equipped with an FRA32M frequency response analyser, connected to an LED driver kit which powered a $470 \mathrm{~nm}$ high-power LED. The light intensity was modulated by $10 \%$ between $100 \mathrm{kHz}$ and $0.1 \mathrm{~Hz}$.

\section{Results and discussion}

Fig. 3 gives an overview of the impact of Sn-doping on the film morphology. The SEM top view images show a disordered mesoporous "worm-like" structure for films prepared with up to $20 \%$ Sn-precursor, similar to that seen for various hematite 


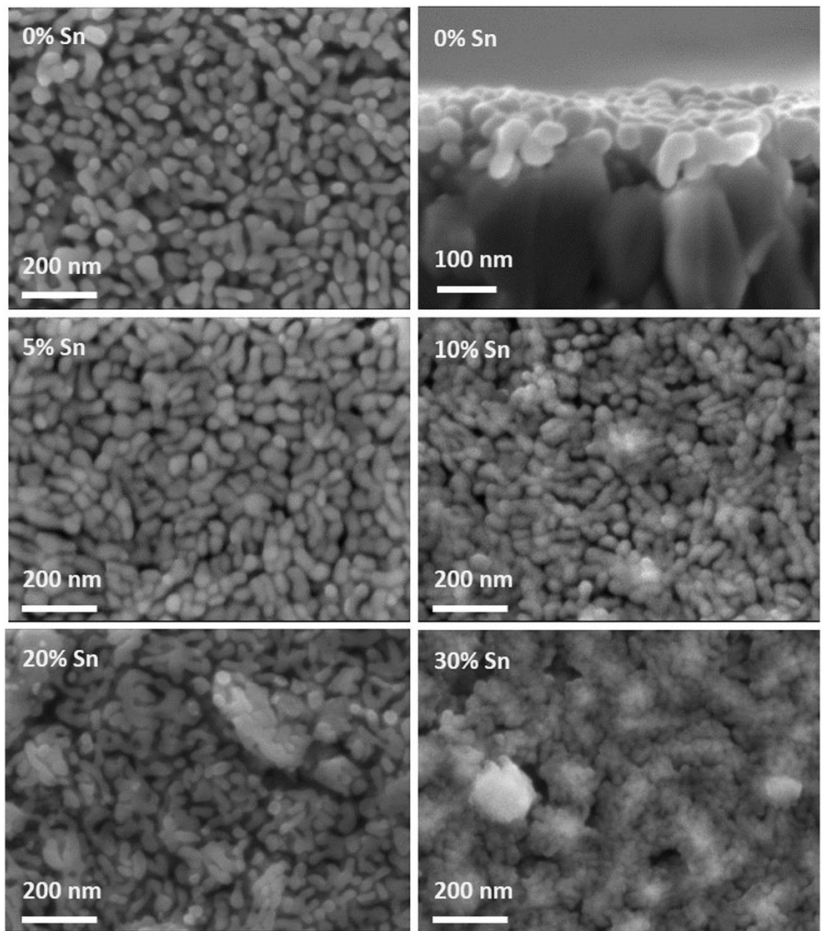

Fig. 3 Top view and cross section SEM images of undoped hematite films (top). Top view SEM-image of films prepared with 5\%, 10\%, 20\% and 30\% $\mathrm{Sn}$-precursor in the hematite synthesis. Each item is labelled with the appropriate Sn-precursor percentage; all films are on an FTO coated glass substrate.

films prepared by other solution-based synthetic routes. ${ }^{11,13,14,53,54}$ The morphology remains practically unchanged with the addition of Sn-precursor to the hematite synthesis, with a mean particle size slightly decreasing from $c a .35 \times 80 \mathrm{~nm}$ to $c a .30 \times 70 \mathrm{~nm}$ for the films prepared without Sn-precursor and with $20 \%$ Sn-precursor added to the synthesis, respectively. The appearance of protruding FTO features in the $20 \%$ Sn-doped film is discussed further in the ESI. $\uparrow$ The addition of $30 \%$ Sn-precursor leads to a more compact and less well-defined morphology. The cross section SEM view indicates that the film is approximately made up of a monolayer of "worm-like" particles. The roughness factor of the films, of the order of 2.5, was estimated from this description of the morphology, see the ESI. $\dagger$

Fig. 4 compares the steady state current-voltage characteristics of films prepared from precursors containing $0 \% \mathrm{Sn}, 5 \%$ $\mathrm{Sn}, 10 \% \mathrm{Sn}, 20 \% \mathrm{Sn}$ and $30 \% \mathrm{Sn}$. In the case of the pure hematite film, the photocurrent density is very low. For films prepared using precursor solutions with a Sn-content above $5 \%$, the photocurrent increases substantially, reaching a maximum for the $20 \% \mathrm{Sn}$ film. The drop in photocurrent observed for the $30 \%$ Sn film is accompanied by a significant change in morphology, see Fig. 3 . While the slightly smaller feature size of the $20 \%$ Sn sample may contribute to the higher photocurrent, this cannot be the dominant factor, because even at $5 \%$ Sn-precursor, the rise in photocurrent is substantial, although the feature size does not change. The goal of this study was to determine the role played by Sn-doping in improving the PEC performance of hematite during water oxidation. Under standard

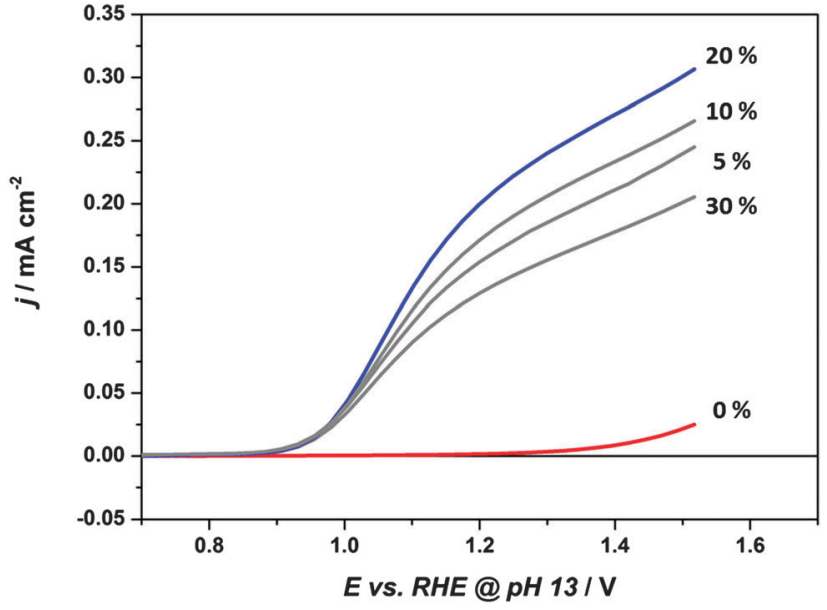

Fig. 4 Current-voltage curves, measured with a scan rate of $20 \mathrm{mV} \mathrm{s}^{-1}$, for single layers of mesoporous hematite prepared with $0 \%, 5 \%, 10 \%, 20 \%$ and $30 \% \mathrm{Sn}$-precursor added to the synthesis. Electrolyte: $0.1 \mathrm{M} \mathrm{NaOH}$. Illumination was through the substrate. $\lambda=455 \mathrm{~nm}$, incident photon flux $=$ $10^{17} \mathrm{~cm}^{-2} \mathrm{~s}^{-1}$. In all cases, the dark current was negligible over the potential range (see Fig. S5, ESI† for dark current voltage curves).

operating conditions, these films have an IQE of the order of 3\%, in close agreement to the IQE of benchmark Si-doped hematite films prepared by APCVD, ${ }^{32}$ see the ESI $\dagger$ for the full analysis. We therefore conclude that these films are suitable model systems for this study.

Improvement in the PEC performance of hematite upon doping are typically attributed to improvements in conductivity. ${ }^{10,11,13,16,18,19,29,30}$ In n-type semiconductors, the conductivity is given by the product $q \mu N_{\mathrm{d}}$, where $\mu$ is the electron mobility. In the case of compact planar electrodes, the doping density (and hence conductivity) can be obtained from Mott Schottky plots according to eqn (5). Rough semiconductor layers, such as the ones studied here, are not ideal for quantitative Mott Schottky analysis, due to the poorly defined surface area, and the possible presence of exposed FTO providing parallel charge transfer pathways. However, assuming the electron mobility, surface area and dielectric constants of the hematite do not change significantly upon doping, a qualitative increase in doping density would be clearly apparent as decrease in the slope of the Mott-Schottky plot. Since there is no significant change in the slopes of the MottSchottky plots (see Fig. S9, ESI $\dagger$ ), we conclude that enhanced conductivity is not responsible for the observed improvements in performance in this system.

In order to elucidate the role of Sn-incorporation in improving the performance of the hematite photoelectrodes, photocurrent transients were recorded, as illustrated in Fig. 5a. In order to ensure that the theoretical treatment outlined above is applicable, the condition that the depletion layer should be narrower than the nanostructure feature size was tested (see ESI $\dagger$ for a detailed discussion of the methods used to evaluate $W_{\mathrm{sc}}$ ). Calculation of the width of the space charge region requires knowledge of the relative permittivity, $\varepsilon_{\mathrm{r}}$, of hematite. Values of $\varepsilon_{\mathrm{r}}$ for hematite in the literature vary considerably. For example Glasscock et $a l .{ }^{55}$ 

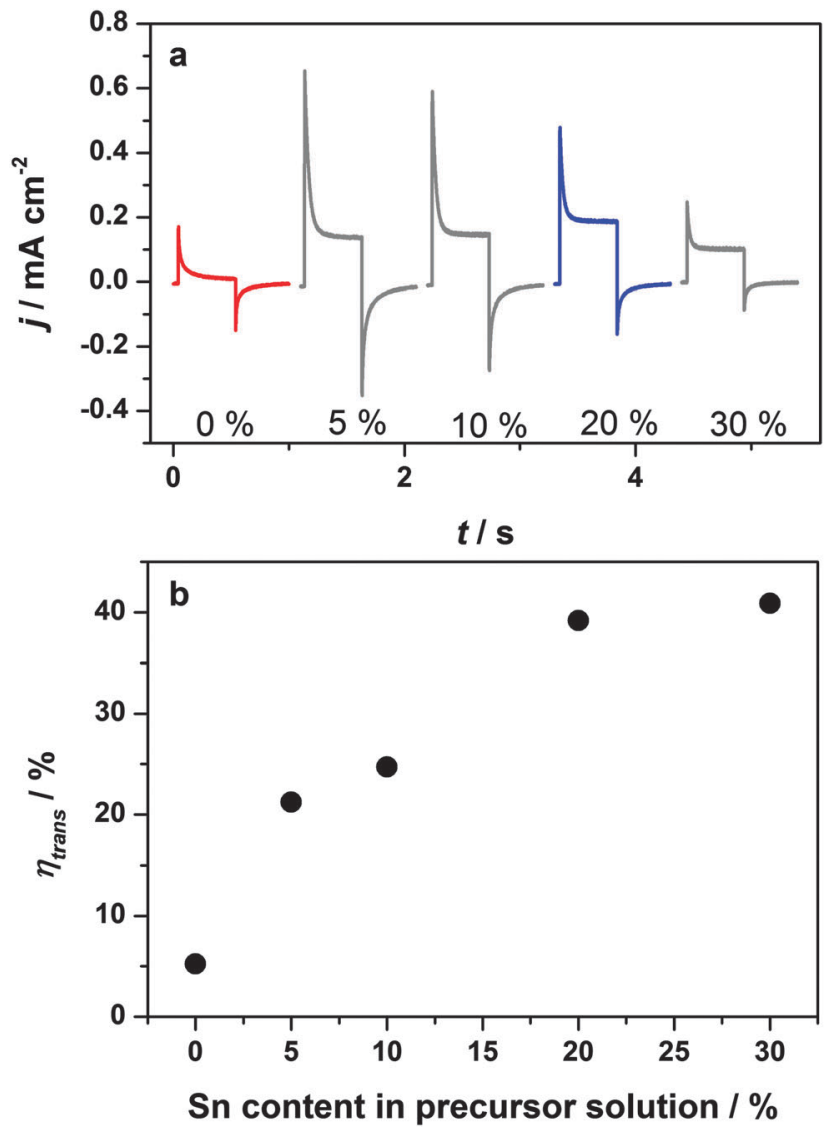

Fig. 5 (a) Photocurrent transients of hematite films prepared with $0 \%, 5 \%$, $10 \%, 20 \%$ and $30 \% \mathrm{Sn}$ precursor in the synthesis. Measured at $1.164 \mathrm{~V}$ vs. RHE under $455 \mathrm{~nm}$ illumination, incident photon flux $10^{17} \mathrm{~cm}^{-2} \mathrm{~s}^{-1}$. (b) Transfer efficiency calculated from the photocurrent transients according to eqn (3).

measured values of $\varepsilon_{\mathrm{r}}$ between 31 and 57, whereas Lunt et al. ${ }^{56}$ have recently calculated values between 7.6 and 26.4 for different crystal orientations and a mean value of around $25 \mathrm{using}$ DFT. At $1.2 \mathrm{~V} v s$. RHE, values of $W_{\text {sc }}$ obtained from the MottSchottky analysis for the $20 \%$ sample in the dark ranged from 10 to $25 \mathrm{~nm}$ for values of relative permittivity of 25 and 57, respectively. By contrast, analysis of the EQE data using eqn (8) indicated much smaller values of the space charge layer thickness in the range of a few $\mathrm{nm}$ (see ESI $\dagger$ for details). This lower value is likely to be due to a reduction of band bending arising from the build-up of holes at the surface, which results in more potential being dropped across the Helmholtz layer rather than across the depletion layer. It follows that - under illumination at least - the condition of a well-defined depletion layer with $W_{\text {sc }}$ smaller than the feature size ( $\geq 30 \mathrm{~nm}$ ) should be satisfied. This reduction in $W_{\mathrm{sc}}$ under illumination highlights why small amplitude perturbation methods such as IMPS are preferable to large amplitude ones such as photocurrent transients. Further evidence for the assumption that $W_{\text {sc }}$ is smaller than the feature size is provided by the fact that the photocurrent does not saturate, but instead increases with potential over the whole potential range. This indicates that the space charge layer continues to penetrate deeper into the film as the applied voltage increases.

The photocurrent transients shown in Fig. 5a clearly indicate that almost all holes reaching the surface of the $0 \% \mathrm{Sn}$ sample recombine, leading to a negligible steady state photocurrent. Addition of only 5\% Sn-precursor to the hematite synthesis increases both the instantaneous and steady state current densities significantly. Since $j_{(t=0)}$ corresponds to the flux of holes swept to the surface upon illumination, an increase in its value indicates an increase in the charge separation efficiency (provided the light harvesting efficiency remains unchanged), suggesting that the "bulk" properties of the material improve. Possible reasons for improved charge separation include a reduction in space charge recombination losses, or an enhanced hole diffusion length. Interestingly, $j_{(t=0)}$ decreases with further addition of $\mathrm{Sn}$. This is most pronounced for the $30 \%$ Sn-doped sample, which has a significantly less well-defined morphology compared to the rest of the series, see Fig. 3. We attribute the slight decrease in $j_{(t=0)}$ for the $20 \%$ sample to a the lower extinction coefficient (and hence light harvesting efficiency) of these samples, see Fig. S4d (ESI $\dagger$ ). This lower extinction coefficient is an artefact related to the film preparation, and is discussed in detail in the ESI. $\dagger$

Most remarkably, the transfer efficiencies, obtained from the ratio $j_{\mathrm{ss}} / j_{(t=0)}$ according to eqn (3), increase steadily with increasing Sn content, see Fig. 5b. The increased transfer efficiency brought about by the incorporation of tin into the hematite can be explained in terms of the competition between interfacial transfer of holes taking part in the OER, and electron-hole recombination at the surface, see eqn (3). On the one hand, the transfer efficiency can be improved by increasing the rate of hole transfer across the interface, the kinetics of which are known to be very slow at hematite photoanodes. $^{57}$ On the other hand, suppression of surface recombination can also significantly enhance performance..$^{7,36}$ The objective of the present study was a clear distinction between these two possibilities.

Although the analysis of photocurrent transients demonstrates that adding tin improves the hole transfer efficiency, further quantitative analysis was not attempted, since "on-off" illumination is likely to change the band bending as a consequence of the build-up of holes at the surface. For this reason, we used IMPS to extract the time constants for hole-transfer and surface electron-hole recombination as described in the theory section. In the following discussion, we focus on the comparison of the pure hematite and 20\% Sn-doped sample, since this gave the highest photocurrent. Typical IMPS spectra obtained for samples doped with $0 \%$ and $20 \%$ Sn are shown in Fig. 6. Both doped and undoped samples give very similar highfrequency semi-circles in the lower quadrant, which correspond to the RC attenuation of the IMPS response with the time constant $R_{\text {series }} C_{\mathrm{sc}}$. Since the series resistance is of the order of $25 \Omega$ for both samples, this indicates that the space charge capacitance, and hence the width of the space charge layer, is not changed by the incorporation of Sn. Since the time constant of the recombination semicircle is at least two orders of 


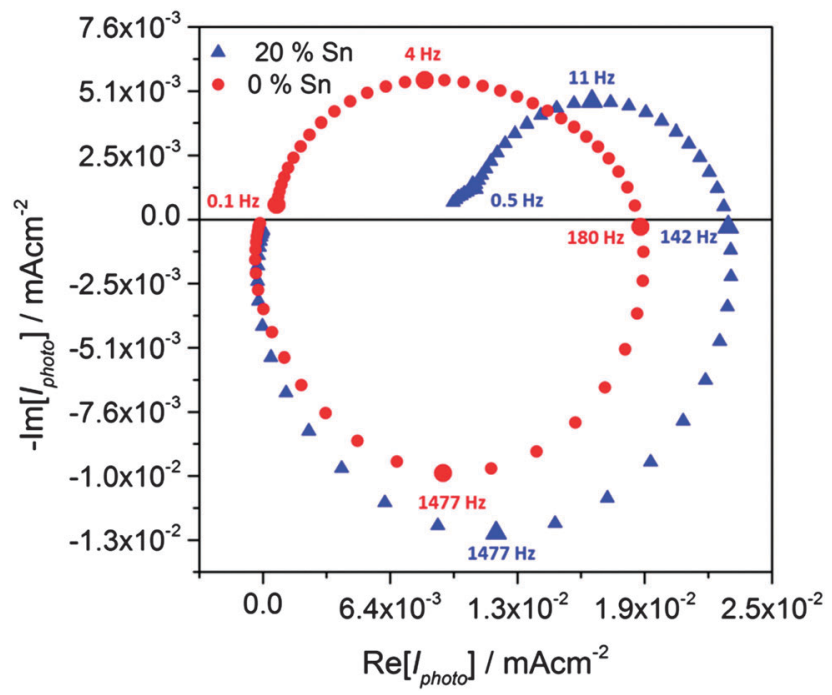

Fig. 6 IMPS spectra of samples prepared with 0\% and 20\% Sn-precursor in the synthesis, recorded at $1.164 \mathrm{~V}$ vs. RHE. $\lambda=470 \mathrm{~nm}$, incident photon flux $10^{17} \mathrm{~cm}^{-2} \mathrm{~s}^{-1}$. Note the large difference in the low frequency semicircles, which reflect the competition between charge transfer and recombination.

magnitude slower than the RC time constant, RC attenuation does not interfere with the kinetic analysis. ${ }^{7}$

By contrast, the low-frequency semicircles in the upper quadrant, which correspond to the competition between charge transfer and recombination, are quite different for the two samples. Whereas the undoped sample gives a semicircle that returns almost to the origin at low frequencies, the semicircle for the Sn-doped sample is much smaller with a low frequency intercept that is much larger. The ratios of the high and low frequency intercepts of the recombination semicircles give values of the transfer efficiency, see Fig. 7a. These values are in excellent agreement with the results obtained from the analysis of the corresponding photocurrent transients, see Fig. $5 \mathrm{~b}$. The potential dependence of $k_{\text {trans }}$ and $k_{\text {rec }}$ obtained from the analysis of the IMPS responses is illustrated in Fig. $7 \mathrm{~b}$ and $\mathrm{c}$. The $k_{\text {rec }}$ values are very similar for both samples, except at the most negative potentials, where the $20 \%$ Sn sample exhibits slightly faster recombination. Strikingly, Sn-inclusion increases $k_{\text {trans }}$ by more than an order of magnitude across almost the whole potential range, indicating better catalysis of the light-driven OER on the surface of tin-doped hematite (note the logarithmic scale in Fig. 7c).

Having established that the improved performance of hematite photoanodes prepared with an additional Sn-precursor in the synthesis is due to the better catalysis of the OER reaction, we investigated the location of the $\mathrm{Sn}$ in the structure. Powder XRD and electron diffraction in TEM mode (Fig. S1 and S3 in the ESI $\dagger$ ) indicate that the overall structure of the material remains that of hematite, without the formation of secondary phases $\left(\mathrm{SnO}_{2}\right.$, for example). The presence of $\mathrm{Sn}$ in the hematite layers was confirmed by EDX in TEM mode. 25 individual EDX spectra were acquired on $20 \%$ Sn-doped samples, for which both the position of the electron beam and its diameter were

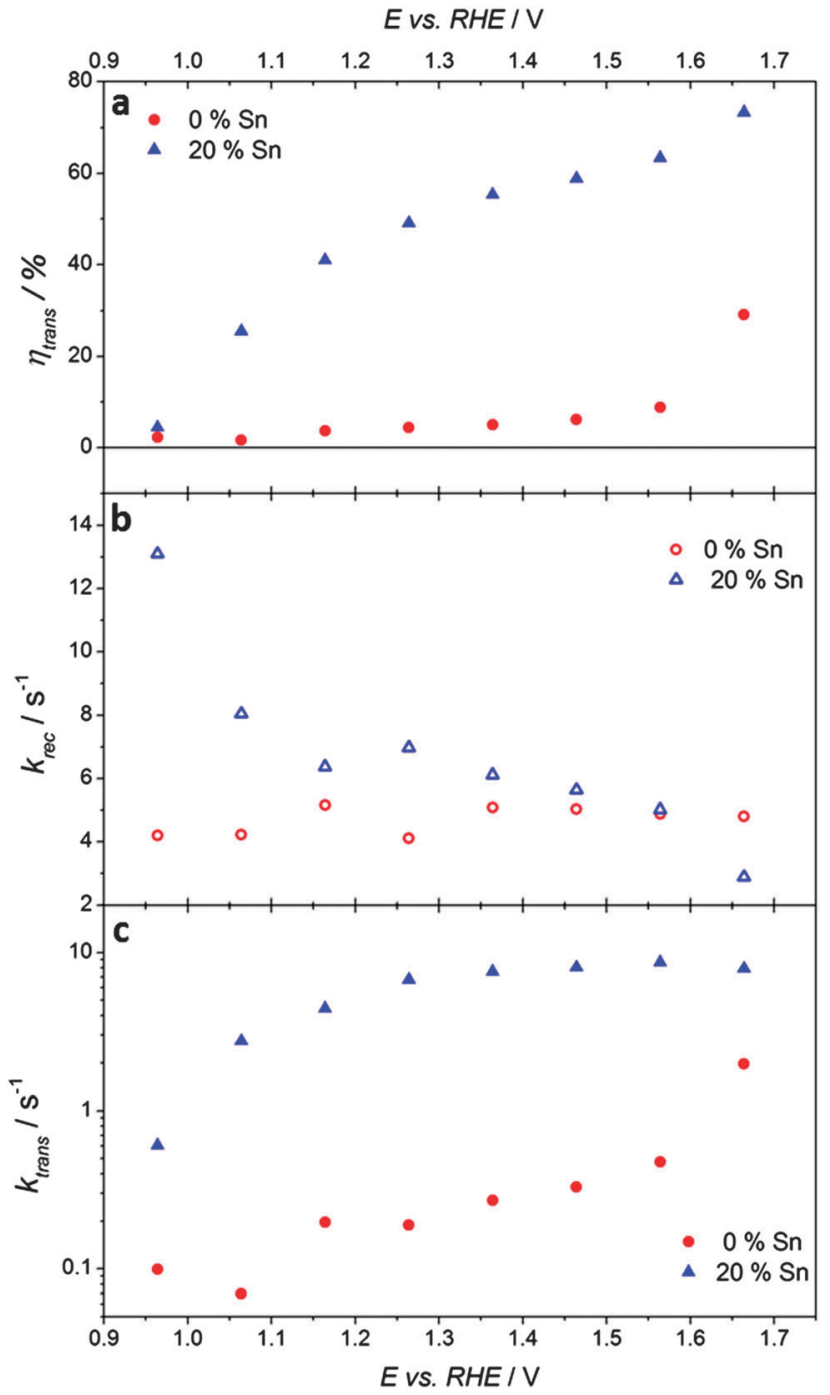

Fig. 7 Parameters extracted from IMPS of photoanodes prepared with $0 \%$ and $20 \% \mathrm{Sn}$-precursor in the synthesis, illuminated by a $470 \mathrm{~nm}$ LED, intensity $10^{17} \mathrm{~cm}^{-2} \mathrm{~s}^{-1}$. (a) Transfer efficiency, (b) rate constant for electron-hole recombination, (c) rate constant for hole transfer.

chosen at random, leading to EDX-measurements of areas of several micrometers as well as of individual particles. All measurements yielded similar Sn contents, indicating a homogeneous distribution on the micron scale. A mean Sn-concentration of $3.2 \pm 1.5$ atomic \% was calculated using the Cliff-Lorimer equation.

Since these measurements did not probe the distribution of Sn within the individual hematite particles, scanning transmission electron microscopy (STEM) was employed in combination with EDX to probe the Sn-content with a step size of approximately $2 \mathrm{~nm}$. To this end, an electron beam with a diameter of less than $1 \mathrm{~nm}$, and consequently low intensity, was used. Line scans across the width of a particle can reveal inhomogeneities between surface and bulk compositions, as illustrated in the inset of Fig. 8b. Close to the edge of the particle, when the STEM beam is tangent to the particle edge, the electron beam probes primarily the surface. In contrast, 

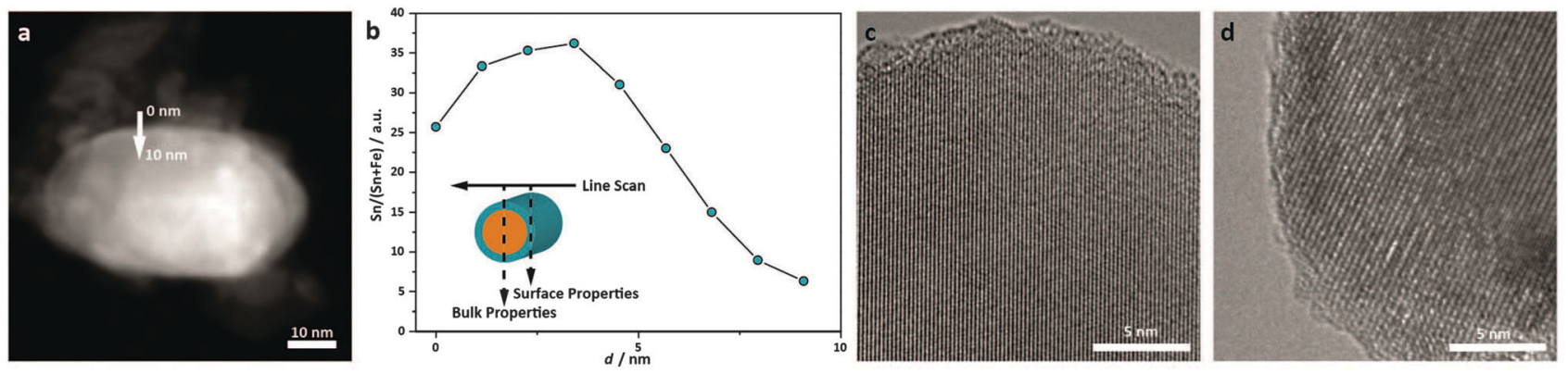

Fig. 8 (a) A HAADF-STEM image of the $20 \%$ Sn particles probed with EDX and (b) relative atomic content of Sn to total metal atomic content as a function of position along the line-scan marked in (a). The sketch inset in (b) illustrates how the STEM beam probes the bulk and surface properties of the particles. Furthermore, HRTEM images of (c) undoped, and (d) Sn-doped, hematite are shown. In (c), the (202) lattice planes are shown, in (d) the (012) lattice planes. In both images, a distortion from the perfect lattice is visible at the surface, indicating atomic disorder in the outer 5 Angstroms.

when the beam is incident normal to the surface of the particle, the X-ray generated due to inelastic scattering of the electron beam is dominated by the bulk (X-rays stemming from the surface contribute to a lesser extent to the total signal, due to the shorter path length through the surface with respect to the bulk). Six particles were probed along lines approximately perpendicular to the surface, such as the one depicted in Fig. 8a. Due to an insufficient signal-to-noise-ratio, background subtraction could not be performed, and therefore the local atomic ratio could not be quantified. However, the ratio of the intensity of the signals attributable to Sn and that obtained from both $\mathrm{Sn}$ and Fe (including the background), revealed a substantial Sn-enrichment at all measured surfaces. The Sn-content strongly decays towards the middle of the particle, where very little signal attributable to Sn was detected. The enrichment of Sn at the surface is also visible in the HAADFSTEM images as a white rim. Since the signal in HAADF-STEM images is approximately proportional to the square of the atomic number for a given thickness ${ }^{58}$ this bright rim is attributed to a higher average atomic number, which is consistent with the inclusion of Sn in the surface atomic columns. Thus, we conclude from our EDX line scan and HAADF-STEM results that the introduction of a Sn-precursor into the hematite synthesis leads to a gradient doping with preferential incorporation of Sn-atoms near the surface of the hematite nanoparticles. Due to the small overall content of Sn, an investigation of the mode of tin incorporation into the hematite structure proved difficult. However, in HRTEM images such as those shown in Fig. $8 \mathrm{c}$ and d, the lattice planes of several dozen undoped and doped particles extend to the surface, and there is no evidence for newly formed separate phases such as $\mathrm{SnO}_{2}$ or $\mathrm{SnO}$ at the surface. We therefore conclude that the Sn incorporates into the hematite structure without substantial structural changes.

Our experimental evidence reveals a structure-function relationship between preferential Sn-doping near the surface of hematite, and an increased hole transfer efficiency (i.e. a higher proportion of photogenerated holes taking part in the OER). This result is in very good agreement with recent reports that Ti- and Si-doping can increase the transfer efficiency in hematite photoanodes during water oxidation. ${ }^{17,31}$ In this work, the power of dynamic light-perturbation techniques further allows us to provide the first demonstration that the increased transfer efficiency brought about by Sn-doping hematite is due to enhanced catalysis of the OER rather than a passivation of surface recombination.

While it is not clear at present how the Sn dopants beneficially impact the OER kinetics, the insight that dopants can speed up the sluggish OER is an important milestone in the optimisation of photoanodes for water oxidation. Indeed, if the specific role of different dopants (and other surface treatments) can be unambiguously identified by methods such as IMPS, the virtues of each could potentially be combined to further improve the efficiency of state of the art hematite photoanodes for water oxidation. Our observations may support recent theoretical predictions that mixed metal sites at the hematite surface (i.e. surface doping) could play a role in balancing the intermediate energetic barriers involved in the OER. ${ }^{59,60}$ Although these studies considered many common hematite dopants such as $\mathrm{Si}$ and $\mathrm{Ti}$, Sn was not included. Given the phenomenological nature of the rate constants obtained here, it is plausible that Sn atoms at the hematite surface may affect the OER intermediates, which would, in turn lead to an increased $k_{\text {trans. }}$. A parallel can be drawn between our findings and recent work by Riha et al., ${ }^{35}$ who suggest that a submonolayer Co-coating also catalyses the OER on hematite photoanodes. Interestingly, the authors note that the existence of neighbouring Fe and Co sites may be crucial to this catalytic activity, which could be an example of the behaviour predicted by Busch and Carter. ${ }^{59,60}$

\section{Conclusions}

We have synthesized thin Sn-doped hematite layers to investigate the role of Sn-doping under light-driven water oxidation conditions. The material is a useful model system because the morphology remains unchanged upon doping and the internal quantum efficiency is similar to that reported for benchmark hematite photoanodes prepared by APCVD and ultrasonic spray pyrolysis. Transient photocurrent responses to chopped illumination revealed that the efficiency of hole-transfer to the solution phase during the OER increased significantly due to 
the Sn-doping. A study by IMPS revealed a tenfold increase in the rate of hole-transfer, i.e. the OER appears catalysed on the Sn-doped hematite as compared to plain hematite. While the mechanism by which the Sn dopant influences the surface kinetics is not yet known, we report the first example of a dopant catalysing the OER on hematite. STEM-EDX line scans revealed a Sn-enrichment at the surface of the nanoparticles, indicating a structure-function relationship between the surface nature of the Sn-doping, and the improved catalytic properties at the surface. While all dopants may not affect hematite in this way, catalysis due to surface doping could be a more widespread effect than currently realized. Application of techniques such as IMPS to distinguish between changes in the rates of hole transfer and surface recombination brought about by inclusion of dopant atoms in other cases would therefore be useful. The combination of dopants playing complementary roles in the enhancement of hematite photoanodes for water oxidation could be a useful strategy towards significant future improvements in performance.

\section{Acknowledgements}

The work was supported by the German Research Foundation, DFG (SPP 1613), Nanosystems Initiative Munich (NIM) and LMUexcellent funded by the DFG, the Bavarian research network 'Solar Technologies Go Hybrid' and UMWELTnanoTECH, the Center for NanoScience (CeNS) and LMUmentoring. Ilina Kondofersky is gratefully acknowledged for insightful discussions. We also thank Steffen Schmidt and Florian Auras for SEM measurements.

\section{References}

1 J. R. Bolton, S. J. Strickler and J. S. Connolly, Nature, 1985, 316, 495-500.

2 A. J. Nozik, Appl. Phys. Lett., 1976, 29, 150-153.

3 R. van de Krol, Y. Liang and J. Schoonman, J. Mater. Chem., 2008, 18, 2311-2320.

4 F. F. Abdi, N. Firet and R. van de Krol, ChemCatChem, 2013, 5, 490-496.

5 J. A. Seabold and K.-S. Choi, Chem. Mater., 2011, 23, 1105-1112.

6 A. Paracchino, V. Laporte, K. Sivula, M. Graetzel and E. Thimsen, Nat. Mater., 2011, 10, 456-461.

7 L. M. Peter, K. G. U. Wijayantha and A. Tahir, Faraday Discuss., 2012, 155, 309-322.

8 I. Cesar, A. Kay, J. A. G. Martinez and M. Graetzel, J. Am. Chem. Soc., 2006, 128, 4582-4583.

9 J. Y. Kim, G. Magesh, D. H. Youn, J.-W. Jang, J. Kubota, K. Domen and J. S. Lee, Sci. Rep., 2013, 3, 2681.

10 N. T. Hahn and C. B. Mullins, Chem. Mater., 2010, 22, 6474-6482.

11 C. D. Bohn, A. K. Agrawal, E. C. Walter, M. D. Vaudin, A. A. Herzing, P. M. Haney, A. A. Talin and V. A. Szalai, J. Phys. Chem. C, 2012, 116, 15290-15296.
12 J. Frydrych, L. Machala, J. Tucek, K. Siskova, J. Filip, J. Pechousek, K. Safarova, M. Vondracek, J. H. Seo, O. Schneeweiss, M. Graetzel, K. Sivula and R. Zboril, J. Mater. Chem., 2012, 22, 23232-23239.

13 Y. Ling, G. Wang, D. A. Wheeler, J. Z. Zhang and Y. Li, Nano Lett. , 2011, 11, 2119-2125.

14 K. Sivula, R. Zboril, F. Le Formal, R. Robert, A. Weidenkaff, J. Tucek, J. Frydrych and M. Graetzel, J. Am. Chem. Soc., 2010, 132, 7436-7444.

15 A. Kay, I. Cesar and M. Graetzel, J. Am. Chem. Soc., 2006, 128, 15714-15721.

16 S. Saremi-Yarahmadi, K. G. U. Wijayantha, A. A. Tahir and B. Vaidhyanathan, J. Phys. Chem. C, 2009, 113, 4768-4778.

17 W. D. Chemelewski, N. T. Hahn and C. B. Mullins, J. Phys. Chem. C, 2012, 116, 5256-5262.

18 M. A. Lukowski and S. Jin, J. Phys. Chem. C, 2011, 115, 12388-12395.

19 R. Franking, L. S. Li, M. A. Lukowski, F. Meng, Y. Z. Tan, R. J. Hamers and S. Jin, Energy Environ. Sci., 2013, 6, 500-512.

20 P. Zhang, A. Kleiman-Shwarsctein, Y.-S. Hu, J. Lefton, S. Sharma, A. J. Forman and E. McFarland, Energy Environ. Sci., 2011, 4, 1020-1028.

21 G. Wang, Y. Ling, D. A. Wheeler, K. E. N. George, K. Horsley, C. Heske, J. Z. Zhang and Y. Li, Nano Lett., 2011, 11, 3503-3509.

22 X. Lian, X. Yang, S. Liu, Y. Xu, C. Jiang, J. Chen and R. Wang, Appl. Surf. Sci., 2012, 258, 2307-2311.

23 J. Deng, J. Zhong, A. Pu, D. Zhang, M. Li, X. Sun and S.-T. Lee, J. Appl. Phys., 2012, 112, 0843121-0843126.

24 Y.-S. Hu, A. Kleiman-Shwarsctein, A. J. Forman, D. Hazen, J.-N. Park and E. W. McFarland, Chem. Mater., 2008, 20, 3803-3805.

25 A. Mao, N.-G. Park, G. Y. Han and J. H. Park, Nanotechnology, 2011, 22, 175703-175710.

26 A. Kleiman-Shwarsctein, Y.-S. Hu, A. J. Forman, G. D. Stucky and E. W. McFarland, J. Phys. Chem. C, 2008, 112, 15900-15907.

27 S. Kumari, C. Tripathi, A. P. Singh, D. Chauhan, R. Shrivastav, S. Dass and V. R. Satsangi, Curr. Sci., 2006, 91, 1062-1064.

28 S. U. M. Khan and Z. Y. Zhou, J. Electroanal. Chem., 1993, 357, 407-420.

29 I. Cesar, K. Sivula, A. Kay, R. Zboril and M. Graetzel, J. Phys. Chem. C, 2009, 113, 772-782.

30 P. Liao, M. C. Toroker and E. A. Carter, Nano Lett., 2011, 11, 1775-1781.

31 O. Zandi, B. M. Klahr and T. W. Hamann, Energy Environ. Sci., 2013, 6, 634-642.

32 H. Dotan, K. Sivula, M. Graetzel, A. Rothschild and S. C. Warren, Energy Environ. Sci., 2011, 4, 958-964.

33 S. D. Tilley, M. Cornuz, K. Sivula and M. Graetzel, Angew. Chem., Int. Ed., 2010, 49, 6405-6408.

34 B. Klahr, S. Gimenez, F. Fabregat-Santiago, J. Bisquert and T. W. Hamann, J. Am. Chem. Soc., 2012, 134, 16693-16700.

35 S. C. Riha, B. M. Klahr, E. C. Tyo, S. Seifert, S. Vajda, M. J. Pellin, T. W. Hamann and A. B. F. Martinson, ACS Nano, 2013, 7, 2396-2405. 
36 M. Barroso, A. J. Cowan, S. R. Pendlebury, M. Graetzel, D. R. Klug and J. R. Durrant, J. Am. Chem. Soc., 2011, 133, 14868-14871.

37 L. M. Peter, Chem. Rev., 1990, 90, 753-769.

38 L. M. Peter, E. A. Ponomarev and D. J. Fermin, J. Electroanal. Chem., 1997, 427, 79-96.

39 E. A. Ponomarev and L. M. Peter, J. Electroanal. Chem., 1995, 396, 219-226.

40 E. A. Ponomarev and L. M. Peter, J. Electroanal. Chem., 1995, 397, 45-52.

41 H. Tang, M. A. Matin, H. Wang, T. Deutsch, M. Al-Jassim, J. Turner and Y. Yan, J. Appl. Phys., 2011, 110.

42 W. W. Gärtner, Phys. Rev., 1959, 116, 84.

43 M. P. Dareedwards, J. B. Goodenough, A. Hamnett and P. R. Trevellick, J. Chem. Soc., Faraday Trans. 1, 1983, 79, 2027-2041.

44 C. Y. Cummings, F. Marken, L. M. Peter, A. A. Tahir and K. G. U. Wijayantha, Chem. Commun., 2012, 48, 2027-2029.

45 L. M. Peter, J. Solid State Electrochem., 2013, 17, 315-326.

46 K. G. U. Wijayantha, S. Saremi-Yarahmadi and L. M. Peter, Phys. Chem. Chem. Phys., 2011, 13, 5264-5270.

47 L. M. Peter and D. Vanmaekelbergh, in Adv. Electrochem. Sci. Eng., ed. R. C. K. Alkire and D. M. Kolb, Weinheim, 1999, vol. 6, pp. 77-163.

48 L. M. Peter and H. Tributsch, in Nanostructured And Photoelectrochemical Systems For Solar Photon Conversion, ed. M. D. Archer and A. J. Nozik, Imperial College Press, London, 2008, vol. 3, pp. 675-736.
49 D. J. Fermin, E. A. Ponomarev and L. M. Peter, J. Electroanal. Chem., 1999, 473, 192-203.

50 S. R. Morrison, Electrochemistry at Semiconductor and Oxidized Metal Electrodes, Plenium Press, 1980.

51 E. Redel, P. Mirtchev, C. Huai, S. Petrov and G. A. Ozin, ACS Nano, 2011, 5, 2861-2869.

52 B. M. Klahr, A. B. F. Martinson and T. W. Hamann, Langmuir, 2011, 27, 461-468.

53 R. Morrish, M. Rahman, J. M. D. MacElroy and C. A. Wolden, ChemSusChem, 2011, 4, 474-479.

54 W. Hamd, S. Cobo, J. Fize, G. Baldinozzi, W. Schwartz, M. Reymermier, A. Pereira, M. Fontecave, V. Artero, C. Laberty-Robert and C. Sanchez, Phys. Chem. Chem. Phys., 2012, 14, 13224-13232.

55 J. A. Glasscock, P. R. F. Barnes, I. C. Plumb, A. Bendavid and P. J. Martin, Thin Solid Films, 2008, 516, 1716-1724.

56 R. Lunt, A. Jackson and A. Walsh, Chem. Phys. Lett., 2013, 586, 67-69.

57 M. Barroso, C. A. Mesa, S. R. Pendlebury, A. J. Cowan, T. Hisatomi, K. Sivula, M. Graetzel, D. R. Klug and J. R. Durrant, Proc. Natl. Acad. Sci. U. S. A., 2012, 109, 15640-15645.

58 D. B. Williams and B. C. Carter, Transmission Electron Microscopy, Springer, New York, 2009.

59 P. Liao, J. A. Keith and E. A. Carter, J. Am. Chem. Soc., 2012, 134, 13296-13309.

60 M. Busch, E. Ahlberg and I. Panas, Phys. Chem. Chem. Phys., 2011, 13, 15062-15068. 